\title{
Structural OCT Parameters Associated with Treatment Response and Macular Neovascularization Onset in Central Serous Chorioretinopathy
}

\author{
Alessandro Arrigo (D) - Alessandro Calamuneri · Emanuela Aragona • \\ Alessandro Bordato - Alessio Grazioli Moretti · Alessia Amato • \\ Francesco Bandello · Maurizio Battaglia Parodi
}

Received: December 29, 2020 / Accepted: January 29, 2021 / Published online: February 19, 2021

(C) The Author(s) 2021

\section{ABSTRACT}

Introduction: This study aimed to assess quantitative factors associated with treatment response and macular neovascularization (MNV) onset in central serous chorioretinopathy (CSC) through an artificial intelligencebased approach.

Methods: The study was designed as an interventional, prospective case series with a planned follow-up of 36 months. We included only eyes demonstrating the first episode of CSC. All the patients underwent eplerenone or photodynamic therapy (PDT) treatment. Eyes developing MNV underwent anti-VEGF injections. We developed an artificial intelligence-based model to assess predictive quantitative structural optical coherence tomography (OCT) factors related to treatment response and onset of

Supplementary Information The online version contains supplementary material available at https:// doi.org/10.1007/s40123-021-00336-3.

A. Arrigo $(\bowtie) \cdot$ E. Aragona $\cdot$ A. Bordato ·

A. Grazioli Moretti · A. Amato · F. Bandello .

M. Battaglia Parodi

Department of Ophthalmology, IRCCS Ospedale

San Raffaele, University Vita-Salute, Milan, Italy

e-mail: alessandro.arrigo@hotmail.com

A. Calamuneri

University of Messina, Messina, Italy
MNV. Main outcome measures were best-correct visual acuity (BCVA), central macular thickness (CMT), retinal thickness (RT), retinal pigment epithelium (RPE) thickness, choroidal thickness, Sattler's layer thickness (SLT), Haller's layer thickness, retinal and choroidal hyperreflective foci (HF), and MNV.

Results: We included 96 naïve CSC eyes (96 patients). Baseline BCVA was $0.18 \pm 0.25 \mathrm{log}$ MAR, which increased to $0.16 \pm 0.27 \log$ MAR after 3 years $(p>0.05)$. Baseline CMT was $337 \pm 126 \mu \mathrm{m}$, which improved to $229 \pm 40 \mu \mathrm{m}$ after 3 years $(p<0.01)$. We observed good response to eplerenone in $40 / 78$ (51\%) eyes, whereas 38/78 (49\%) eyes underwent PDT. The artificial intelligence model showed choroidal $\mathrm{HF}$ and age as determining factors of good response to eplerenone or PDT. RPE thickness $<36 \mu \mathrm{m}$, RT $<300 \mu \mathrm{m}$, and SLT $<50 \mu \mathrm{m}$ increased probability of $50 \%$ of having MNV.

Conclusions: CSC response to eplerenone or PDT is influenced by choroidal HF and patient age. RPE and SLT represent relevant factors for onset of MNV.

Keywords: Artificial intelligence; Central serous chorioretinopathy; Eplerenone; OCT; Photodynamic therapy; Treatment response 


\section{Key Summary Points}

Central serous chorioretinopathy (CSC) is a possible cause of visual acuity loss, nowadays managed by oral eplerenone or photodynamic therapy.

There is no consensus in the literature regarding the best treatment strategy; furthermore, recent studies have raised many doubts about the efficacy of eplerenone.

In the present study, we propose quantitative structural optical coherence tomography (OCT) biomarkers in central serous chorioretinopathy, assessed by an artificial intelligence-based approach, associated with response to oral eplerenone or photodynamic therapy.

We have also assessed quantitative biomarkers associated with an increased risk of development of macular neovascularization.

\section{DIGITAL FEATURES}

This article is published with digital features to facilitate understanding of the article. To view digital features for this article go to https://doi. org/10.6084/m9.figshare.13656644.

\section{INTRODUCTION}

Central serous chorioretinopathy (CSC) is a posterior segment disease typically characterized by neurosensory retinal detachment, leading to visual acuity loss [1]. The current management of CSC includes observation, laser application, diuretics, and photodynamic therapy (PDT) [2, 3]. However, response to treatment is highly heterogeneous, and there is as yet no consensus regarding the best therapeutic approach. To date, several papers have reported positive results for both eplerenone and PDT treatment strategies [3-10]. A recent study prompted considerable debate about the suitability of eplerenone oral treatment in the management of CSC, suggesting no indication for this therapeutic choice [11]. However, that trial differed from previous studies supporting the role of eplerenone by also including CSC eyes with macular neovascularization (MNV) [12], together with a lack of a subclassification of the patients. Indeed, in a recent study, our group demonstrated the presence of at least two CSC subtypes, as assessed by quantitative multimodal imaging, associated with different outcomes and risk of MNV onset [13]. Furthermore, some limitations have recently been noted, especially regarding patient selection [14].

The present study investigated the role of quantitative structural optical coherence tomography (OCT) parameters for predicting response to treatment. An artificial intelligencebased approach was adopted to assess whether structural OCT findings may suggest a specific treatment. The same artificial intelligence-based model was further employed to assess the presence of quantitative biomarkers associated with an increased risk of MNV onset.

\section{METHODS}

The study was designed as an interventional, prospective case series with a 36-month followup. Patients presenting with a first, treatmentnaïve episode of CSC were recruited at the Ophthalmology Unit of San Raffaele Hospital, Milan, between January 2015 and October 2016. Signed informed consent was obtained from all patients. The study was conducted in accordance with the Declaration of Helsinki and was approved by the Ethical Committee of VitaSalute San Raffaele University in Milan.

The diagnosis of CSC was made by means of structural OCT and fluorescein angiography (FA)-indocyanine green angiography (ICGA) imaging (Spectralis HRA+OCT; Heidelberg Engineering, Heidelberg, Germany) and OCT angiography (DRI Triton; Topcon, Japan). In particular, clinical evidence of visual acuity loss or distortion, confirmed by the presence of serous subretinal exudation, RPE signal changes on 
structural OCT, and FA/ICGA CSC-related choroidal alterations, was useful in the diagnosis. Exclusion criteria were refractive error greater than $\pm 3 \mathrm{D}$, high media opacity, any other type of retinal or optic nerve diseases, ocular surgery within the last 6 months, or any systemic condition potentially affecting the analyses. The onset of MNV was confirmed by further FA/ICGA examination, eventually supported by OCT-angiography (OCTA).

Ophthalmologic examination included BCVA measurement using standard ETDRS charts, slit-lamp biomicroscopy of the anterior and posterior segments, Goldmann applanation tonometry, FA, ICGA, OCT, and OCTA. All the patients were periodically monitored, at the ophthalmologists' discretion, and guided by the disease course. For all the quantitative analyses, we considered the standardized $\mathrm{T} 0, \mathrm{~T} 1, \mathrm{~T} 2$, and $\mathrm{T} 3$ examinations, where $\mathrm{T} 1, \mathrm{~T} 2$, and $\mathrm{T} 3$ represent the first, second, and third year of followup, respectively.

The structural OCT acquisition protocol included raster, radial, and dense scans with a large number of frames (automatic retinal tracking $[\mathrm{ART}]>25)$ and enhanced depth imaging (EDI). Structural OCT scans measured central macular thickness (CMT) and retinal thickness (RT) at baseline and over the entire 36-month follow-up, while inverted structural OCT color images assessed retinal and choroidal hyperreflective foci (retinal and choroidal HF, respectively). CMT and RT were automatically obtained by the device software. Wishing to offer a clinically applicable evaluation of HF, we did not calculate the entire number of retinal and choroidal $\mathrm{HF}$, but instead considered more than $10 \mathrm{HF}$ within the retina or choroid as positive for HF. Choroidal thickness (CT), measured manually, was considered as the mean obtained from five measurements performed at these points: subfoveal, $750 \mu \mathrm{m}$ (right-left), and $1500 \mu \mathrm{m}$ (right-left). The same steps were adopted for Haller's layer thickness (HLT), while Sattler's layer thickness (SLT) was obtained from the difference between CT and HLT.

All the structural OCT measurements were performed by two independent researchers at least twice to test reproducibility and repeatability (overall 0.89 reproducibility [range $0.85-0.90$ ] and 0.88 repeatability [range $0.86-0.91])$.

All CSC patients started first-line treatment with eplerenone (oral administration $25 \mathrm{mg} /$ day for the first week, increasing to $50 \mathrm{mg} /$ day for up to 4 months if blood serum potassium levels were $5.0 \mathrm{mmol} / \mathrm{L}$ or lower). In the absence of response after 4 months of treatment (clinical response defined as a gain of five or more ETDRS letters; morphological response defined as fluid reduction greater than $20 \%$ on structural OCT), patients were switched to half-dose PDT. CSC patients developing MNV were treated with anti-vascular endothelial growth factor (VEGF) intravitreal injections or PDT, at the ophthalmologist's discretion.

The main outcome was the analysis of the response to treatment leading to subretinal fluid resolution (SRF), adopting an artificial intelligence-based approach based on structural OCT findings.

Factors associated with MNV were investigated by means of a logistic model, in which the presence of MNV (yes or no) was adopted as the dependent variable. The full model consisted of the following variables, sampled at admission (T0): retinal thickness (Tot_Retina), central macular thickness (CMT), highest subretinal fluid thickness (exudate), retinal pigment epithelium (RPE) thickness, choroidal thickness (Tot_Choroid), Sattler's layer thickness (SLT), and Haller's layer thickness (HLT). Age, gender, and disease duration were added as covariates. An automatic procedure was used to determine the best model among the potential explanatory variables. A backward scheme was adopted for this purpose, and the Akaike information criterion (AIC) was employed to identify the optimal model [15].

CSC patients without MNV were studied with regard to the relationship between their treatment and the following explanatory variables measured at T0: Tot_Retina, retinal RPE, Tot_Choroid, retinal hyperreflective foci (HF_ret), and choroidal hyperreflective foci (HF_chor). Sex and gender were included as covariates. To determine the best model, we adopted a logistic model and used the same pipeline as described above. 
We explored ways of choosing the optimal treatment with the aim of finding a potential automatic decision-making process. Adopting a supervised machine learning technique, i.e. a decision tree [16], we entered the following potential predictors in the model: CMT, Tot_Retina, Tot_Choroid, RPE, HF_chor, HF_ret, SLT, HLT, Exudate, Age, Sex.

We analyzed how the morphological parameters recovered longitudinally after treatment. For this objective, we employed a repeated-measures analysis of variance (ANOVA), adding sex and gender as covariates.

The following parameters were used as dependent variables: Tot_Retina, CMT, RPE, Tot_Choroid, SLT, HLT, Exudate, BCVA. To account for assumption violations, we adopted the Greenhouse-Geisser degrees of freedom correction.

All analyses were performed with the R statistical software package, release 3.5.2 [17]. The significance threshold was set at 0.05 , using false discovery rate (FDR) correction when necessary to handle multiple comparison issues. Extensive details regarding the statistical methodology are provided in the Appendix.

\section{RESULTS}

\section{Overall Results}

We recruited 120 eyes of 120 patients affected by a first, treatment-naïve episode of CSC. Twenty-four eyes were excluded for the following reasons: media opacities (20), glaucoma (4). Overall, 96 eyes of 96 treatment-naïve CSC patients (mean age $53 \pm 12$ years; 80 male) were included in the analyses. None of the patients showed MNV at baseline. Over the entire cohort of CSC patients, 18 developed MNV over the 36-month follow-up.

In patients not showing MNV (78/96 eyes), SRF was found to have completely resolved in $55 \%$ of cases at $\mathrm{T} 1,63 \%$ of cases at $\mathrm{T} 2$, and $75 \%$ of cases at T3. Positive treatment response, defined as at least $20 \%$ SRF reduction, was identified in $81 \%$ at $\mathrm{T} 1,90 \%$ at $\mathrm{T} 2$, and $96 \%$ at T3.
Considering the whole CSC group, baseline BCVA was $0.18 \pm 0.25 \log \mathrm{MAR}$, increasing to $0.16 \pm 0.27 \operatorname{logMAR}$ after 3 years $(p>0.05)$; baseline CMT was $337 \pm 126 \mu \mathrm{m}$, improving to $229 \pm 40 \mu \mathrm{m}$ after 3 years $(p<0.01)$. Out of 96 eyes, 18 (19\%) developed MNV; 14/18 (78\%) developed MNV during the follow-up, whereas $4 / 18$ (22\%) showed MNV at the baseline examination.

CSC eyes without MNV responded well to eplerenone in 40/78 (51\%) eyes, whereas 38/78 (49\%) eyes needed to be switched to PDT. Interestingly, of the eyes developing MNV during the follow-up (14/96 eyes, 15\%), 10/14 (71\%) responded well to PDT before MNV onset, whereas $4 / 14$ (29\%) responded well to eplerenone before $\mathrm{MNV}$ onset. In the $\mathrm{MNV}$ group, the mean number of anti-VEGF injections was $7 \pm 4$ over the follow-up; in approximately $60 \%$ of cases, MNV patients underwent additional PDT, with $3 \pm 1$ treatments.

\section{Prediction of the Best Treatment for Patients without MNV}

Complete data are listed in Table 1. The winning model contained the following significant explanatory variables: choroidal HF (e.c. $=2.72$, $p<0.001$ ), retinal HF (e.c. $=-3.11, p<0.001$ ), and age (e.c. $=0.08, p=0.012$ ). The significant age factor reveals a moderate tendency towards better efficacy of PDT over eplerenone when age increases.

Subjects with choroidal HF responded better to PDT $(32 / 47,68 \%)$, while those without choroidal HF responded better to eplerenone $(25 / 31,80 \%)$. Subjects with retinal HF responded better to eplerenone $(36 / 56,64 \%)$, while those without retinal HF responded better to PDT (18/22, 81\%) (Fig. 2). A more complete scenario can be seen in Table 2, which records all combinations of retinal HF and choroidal HF (Fig. 1). It is striking that the presence of only one of the two signs determines the decision, with eplerenone working better with retinal HF and without choroidal HF, whereas PDT was preferred in the reverse circumstances. A more homogeneous distribution can instead be observed when both signs are present or absent. 
Table 1 Quantitative analyses in CSC treatment subgroups

\begin{tabular}{|c|c|c|c|}
\hline & Eplerenone group & PDT group & MNV group \\
\hline Group & 1 & 2 & 3 \\
\hline Number of patients & 40 & 38 & 18 \\
\hline BCVA baseline & $0.1 \pm 0.11$ & $0.21 \pm 0.33$ & $0.33 \pm 0.18$ \\
\hline BCVA 3-year F/U & $0.06 \pm 0.12$ & $0.1 \pm 0.18$ & $0.48 \pm 0.41$ \\
\hline$p$ value & 0.11 & 0.09 & 0.16 \\
\hline CMT baseline $(\mu \mathrm{m})$ & $374 \pm 139$ & $329 \pm 123$ & $271 \pm 66$ \\
\hline CMT 3-year F/U $(\mu \mathrm{m})$ & $225 \pm 39$ & $231 \pm 45$ & $235 \pm 35$ \\
\hline$p$ value & $<0.01$ & $<0.01$ & 0.04 \\
\hline RT baseline $(\mu \mathrm{m})$ & $458 \pm 120$ & $437 \pm 78$ & $387 \pm 94$ \\
\hline RT 3-year F/U $(\mu \mathrm{m})$ & $340 \pm 47$ & $342 \pm 46$ & $361 \pm 60$ \\
\hline$p$ value & $<0.01$ & $<0.01$ & 0.33 \\
\hline CT baseline $(\mu \mathrm{m})$ & $416 \pm 101$ & $419 \pm 91$ & $366 \pm 107$ \\
\hline CT 3-year F/U $(\mu \mathrm{m})$ & $406 \pm 99$ & $395 \pm 85$ & $343 \pm 125$ \\
\hline$p$ value & 0.65 & 0.25 & 0.56 \\
\hline SLT baseline $(\mu \mathrm{m})$ & $117 \pm 39$ & $115 \pm 31$ & $49 \pm 12$ \\
\hline SLT 3-year F/U $(\mu \mathrm{m})$ & $108 \pm 32$ & $111 \pm 28$ & $45 \pm 12$ \\
\hline$p$ value & 0.26 & 0.63 & 0.38 \\
\hline HLT baseline $(\mu \mathrm{m})$ & $298 \pm 81$ & $304 \pm 72$ & $317 \pm 108$ \\
\hline HLT 3-year F/U $(\mu \mathrm{m})$ & $297 \pm 77$ & $284 \pm 64$ & $298 \pm 123$ \\
\hline$p$ value & 0.95 & 0.20 & 0.62 \\
\hline$p$ value & 1 vs. 2 & 1 vs. 3 & 2 vs. 3 \\
\hline BCVA baseline & 0.148 & 0.003 & 0.208 \\
\hline BCVA 3-year F/U & $>0.05$ & $<0.01$ & $<0.01$ \\
\hline CMT baseline $(\mu \mathrm{m})$ & 0.332 & 0.01 & 0.289 \\
\hline CMT 3-year F/U $(\mu \mathrm{m})$ & $>0.05$ & $>0.05$ & $>0.05$ \\
\hline RT baseline $(\mu \mathrm{m})$ & $>0.05$ & 0.04 & 0.261 \\
\hline RT 3-year F/U ( $\mu \mathrm{m})$ & $>0.05$ & 0.420 & 0.512 \\
\hline CT baseline $(\mu \mathrm{m})$ & $>0.05$ & 0.237 & 0.197 \\
\hline CT 3-year F/U $(\mu \mathrm{m})$ & $>0.05$ & 0.087 & 0.214 \\
\hline SLT baseline $(\mu \mathrm{m})$ & $>0.05$ & $<0.01$ & $<0.01$ \\
\hline SLT 3-year F/U $(\mu \mathrm{m})$ & $>0.05$ & $<0.01$ & $<0.01$ \\
\hline HLT baseline $(\mu \mathrm{m})$ & $>0.05$ & $>0.05$ & $>0.05$ \\
\hline
\end{tabular}


Table 1 continued

\begin{tabular}{lccr}
\hline $\boldsymbol{p}$ value & 1 vs. 2 & 1 vs. 3 & 2 vs. 3 \\
\hline HLT 3-year F/U $(\mu \mathrm{m})$ & $>0.05$ & $>0.05$ & $>0.05$
\end{tabular}

The eplerenone group is characterized by good response to eplerenone alone. The PDT group is characterized by poor response to eplerenone, thus requiring the switch to PDT. The MNV group consists of eyes belonging to either the eplerenone or PDT group that developed neovascular complication during the follow-up. Abbreviations: CSC central serous chorioretinopathy, $P D T$ photodynamic therapy, $M N V$ macular neovascularization, $B C V A$ best-corrected visual acuity, $F / U$ follow-up, $C M T$ central macular thickness, $R T$ retinal thickness, $C T$ choroidal thickness, SLT Sattler's layer thickness, HLT Haller's layer thickness

Table 2 Frequency of best treatment according to the presence of retinal HF and choroidal HF

\begin{tabular}{|c|c|c|c|c|c|c|c|c|}
\hline & HFC $=0$ & HFR $=0$ & $\mathrm{HFC}=0$ & HFR $=1$ & $\mathrm{HFC}=1$ & HFR $=0$ & $\mathrm{HFC}=1$ & $\mathrm{HFR}=1$ \\
\hline Eplerenone & 4 & & 21 & & 0 & & 15 & \\
\hline PDT & 4 & & 2 & & 14 & & 18 & \\
\hline $\begin{array}{l}\text { Percentage eplerenone } \\
\text { vs. PDT }\end{array}$ & $(50 / 50)$ & & $(91.3 / 8.7)$ & & $(0 / 100)$ & & $(46 / 54)$ & \\
\hline
\end{tabular}

Percentages are related to intermodal distribution. Abbreviations: $H F C$ hyperreflective foci choroid, $H F R$ hyperreflective foci retina. The presence of HF is expressed as (1), whereas absence of HF is expressed as (0)

The decision-making process forecast by the decision tree is shown in Fig. 2. The presence or absence of choroidal HF is central to the choice. In its absence, the optimal choice in $81 \%$ of cases is eplerenone ( 25 out of 31 cases). In the presence of choroidal HF, the age factor was found to be more relevant, with subjects up to 43 years of age being more successfully treated with eplerenone $(7 / 9,78 \%)$, while for older patients PDT was largely the better choice (30/ $38,79 \%)$.

\section{Macular Neovascularization}

The winning logistic model consisted of the following significant variables: Tot_Retina (estimated coefficient, e.c. $=-0.01872$, $p=0.0373$ ), $\operatorname{RPE}($ e.c. $=1.7546, p=0.020$ ), and SLT (e.c. $=-0.1367, p<0.001)$. Therefore, a decrease in retinal thickness results in a slight increase in the likelihood of encountering
MNV. RPE and SLT thinning were associated with an increased probability of finding MNV. The critical value with regard to RPE thickness was $36 \mu \mathrm{m}$; below this value there was an increased probability of having MNV, while for SLT, the critical value was $50 \mu \mathrm{m}$, and there was an increased probability of up to $50 \%$ of having MNV. As for retinal thickness, a slight increase in probability was observed below $300 \mu \mathrm{m}$. (Fig. 3).

\section{DISCUSSION}

In the present study we analyzed the morphological features of a cohort of patients affected by treatment-naïve CSC, and we identified different subgroups according to their response to specific treatment, either eplerenone or PDT.

CSC eyes with a greater degree of choroidal HF showed a better response to PDT, whereas patients with higher retinal HF responded better 


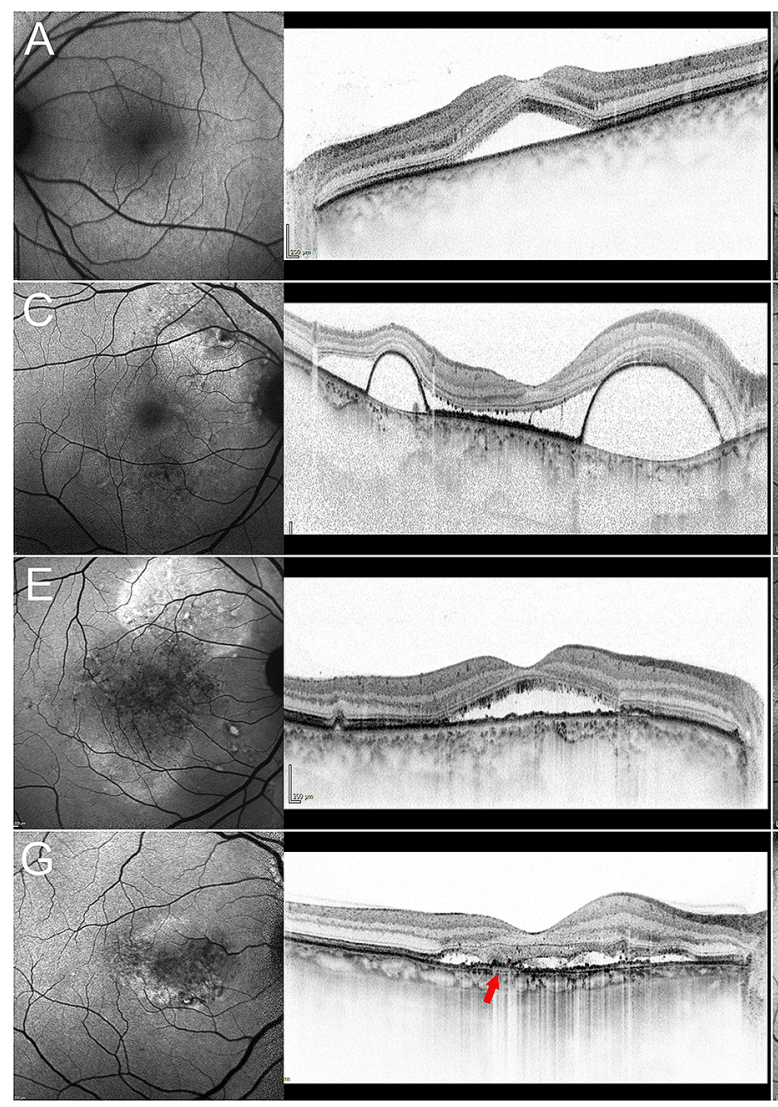

Fig. 1 Hyperreflective foci in CSC. HF may be exclusively retinal (a) or choroidal (c). Patients may also display both retinal and choroidal HF, whether with MNV (red arrow)

to eplerenone. We also found an age effect related to a better response to PDT, which was observed in patients over 43 years of age. The interpretation of the findings is speculative: higher choroidal HF might suggest greater inflammation of the choroid, which would therefore require treatment aimed more directly at the vascular component (e.g. PDT). In contrast, higher retinal HF might suggest an inflammatory reaction involving RPE cells.

$\mathrm{MNV}$ is a vision-threatening complication of CSC $[18,19]$. In the present study we adopted an artificial intelligence-based model to determine whether certain imaging findings might be associated with an increased risk of the onset of MNV. The logistic model detected precise quantitative values associated with MNV. In particular, RPE thickness less than $36 \mu \mathrm{m}$, SLT less than $50 \mu \mathrm{m}$, and RT less than $300 \mu \mathrm{m}$

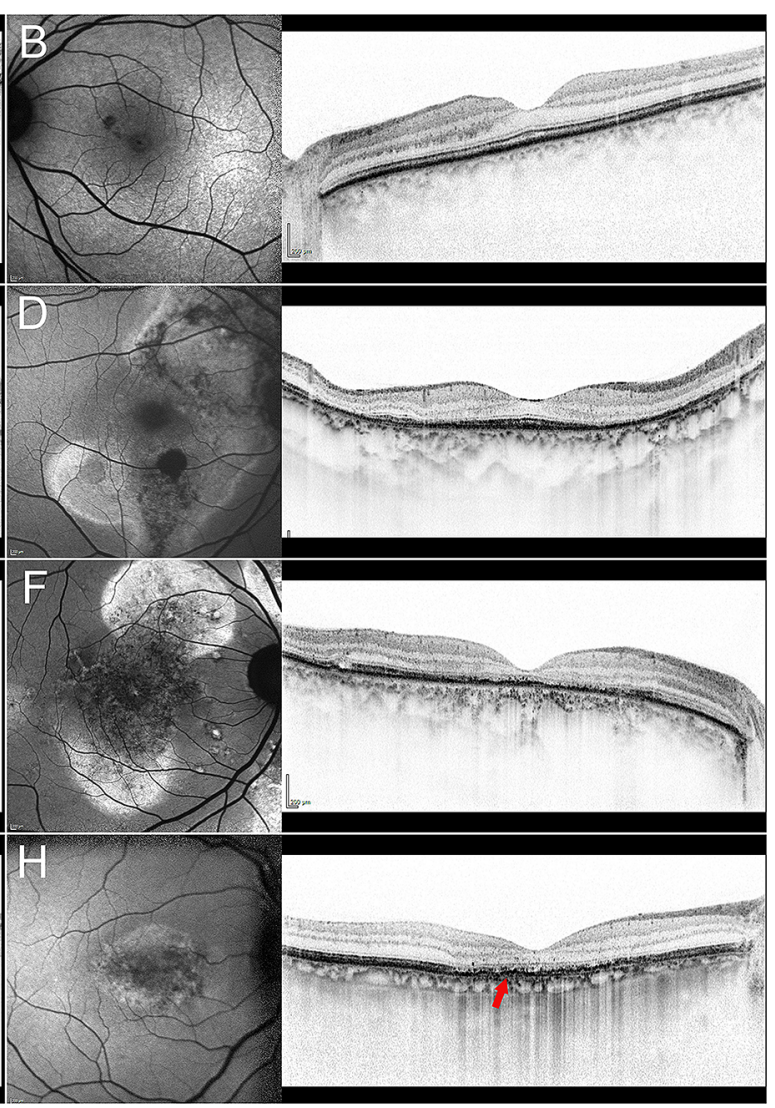

(g) or without MNV (e). The HF localization remains the same immediately after the resolution of the exudation, secondary to the treatment ( $\mathbf{b}, \mathbf{d}, \mathbf{f}, \mathbf{h}$, respectively)

proved to be closely associated with the onset of $\mathrm{MNV}$. The pathogenesis of MNV in CSC is still a matter of debate but includes chronic RPE decompensation and choroidal ischemia [20]. The finding of a greater rate of MNV onset in eyes treated by PDT, with respect to eyes managed only by eplerenone, may suggest that in the balance between vascular and non-vascular dysfunction leading to the onset of CSC, vascular dysregulation might be prominent in eyes requiring PDT, thus justifying the higher probability of developing MNV. Furthermore, considering the treatment planning in the present study, including a first eplerenone treatment and PDT administration only in a second phase, it is possible that the higher chronicity of subretinal exudation in the PDT group may have led to higher RPE occurrence with consequent increased release of pro-angiogenic mediators. 


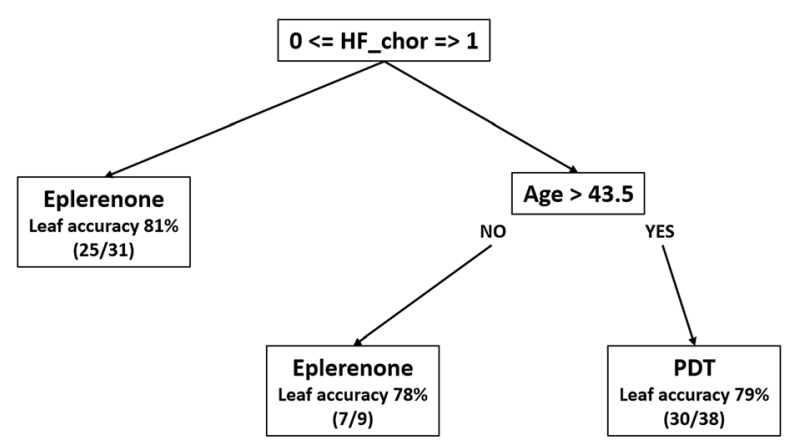

Fig. 2 Decision tree for treatment choice. If the number of choroidal hyperreflective foci $(\mathrm{HF})$ is $<10$, the better response is shown with eplerenone. In the presence of choroidal $\mathrm{HF}>10$, age represents a determinant factor. Patients aged $>43.5$ years show better response to photodynamic therapy, whereas patients aged $<43.5$ years show better response to eplerenone

Our findings are in line with previous theories regarding RPE and choroidal impairment [1], but our analysis provides specific cutoff values that are more effective in identifying CSC patients at risk.
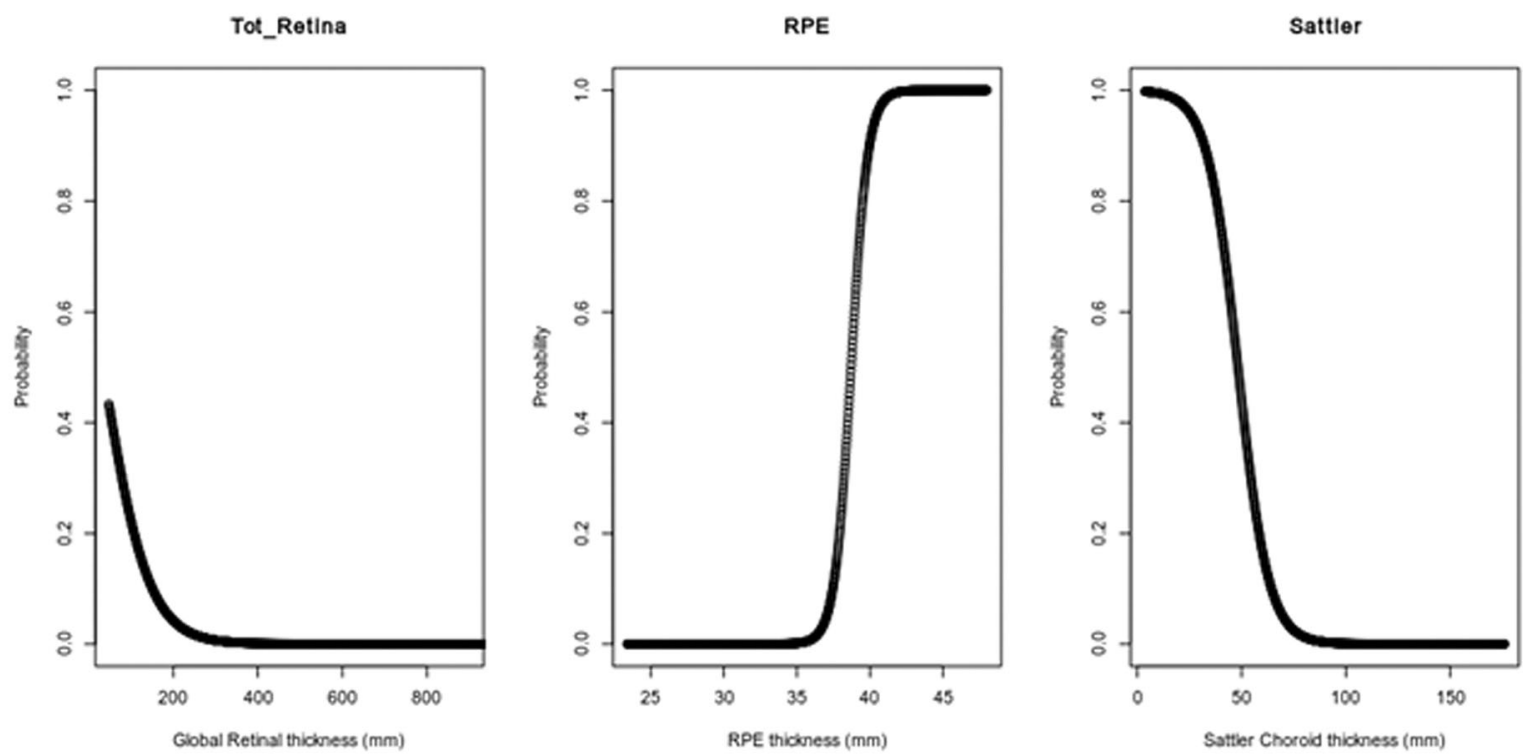

Fig. 3 Simulation of the probability of developing MNV in relation to changes in retinal, RPE and Sattler's layer thickness. The variable of interest was simulated for each panel by including 1000 points, while the other two
The benefit of diuretic therapy has recently been questioned [11], but a possible explanation for the previously described ineffectiveness of eplerenone may lie in the fact that CSC was not broken down into subcategories with different baseline parameters. From this point of view, further investigations including a quantitative categorization of the patients are warranted to draw definite conclusions.

We are aware that our study has several limitations. First, the study was designed considering eplerenone as the first-line treatment, with no control group, and simply replaced with PDT for non-responders. We acknowledge that this choice may have affected the results of the study, but it was supported by previous evidence of efficacy of eplerenone oral treatment, and it also represented an attempt to harmonize the study patients in order to achieve meaningful data. On this basis, further quantitative investigations should be conducted that also include CSC patients managed with a "wait-and-see" strategy. In addition, the number of patients is relatively small to draw definitive conclusions, and the suitability of the 
artificial intelligence model we applied needs to be confirmed in a larger controlled cohort of patients.

\section{CONCLUSION}

In conclusion, this study provides quantitative biomarkers suggestive of good response to eplerenone or PDT. Moreover, we provide defined cutoffs to identify patients at risk of MNV onset. Nevertheless, the present investigation should be viewed as a first project that attempts to identify new biomarkers able to help establish the best therapeutic option in common clinical practice. Indeed, our present findings would benefit from further prospective studies exploring the real usefulness of structural OCT biomarkers in the management of CSC with and without MNV.

\section{ACKNOWLEDGEMENTS}

We thank the participants of the study.

Funding. No funding or sponsorship was received for this study or publication of this article. The Rapid Service Fee was funded by the authors.

Authorship. All named authors meet the International Committee of Medical Journal Editors (ICMJE) criteria for authorship for this article, take responsibility for the integrity of the work as a whole, and have given their approval for this version to be published.

Disclosures. Francesco Bandello: consultant for Alcon (Fort Worth, TX, USA), Alimera Sciences (Alpharetta, GA, USA), Allergan Inc (Irvine, CA, USA), Farmila-Thea (Clermont-Ferrand, France), Bayer Schering Pharma (Berlin, Germany), Bausch and Lomb (Rochester, NY, USA), Genentech (San Francisco, CA, USA), Hoffmann-La Roche (Basel, Switzerland), Novagali Pharma (Évry, France), Novartis (Basel, Switzerland), Sanofi-Aventis (Paris, France), ThromboGenics (Heverlee, Belgium), Zeiss (Dublin, CA, USA). Alessandro Arrigo,
Alessandro Calamuneri, Emanuela Aragona, Alessandro Bordato, Alessio Grazioli Moretti, Alessia Amato, Maurizio Battaglia Parodi have nothing to disclose.

Compliance with ethical guidelines. Signed informed consent was obtained from all patients. The study was conducted in accordance with the Declaration of Helsinki and was approved by the Ethical Committee of Vita-Salute San Raffaele University in Milan.

Data availability. Data sharing is not applicable to this article, as no datasets were generated or analyzed during the current study.

Open Access. This article is licensed under a Creative Commons Attribution-NonCommercial 4.0 International License, which permits any non-commercial use, sharing, adaptation, distribution and reproduction in any medium or format, as long as you give appropriate credit to the original author(s) and the source, provide a link to the Creative Commons licence, and indicate if changes were made. The images or other third party material in this article are included in the article's Creative Commons licence, unless indicated otherwise in a credit line to the material. If material is not included in the article's Creative Commons licence and your intended use is not permitted by statutory regulation or exceeds the permitted use, you will need to obtain permission directly from the copyright holder. To view a copy of this licence, visit http://creativecommons.org/licenses/bync/4.0/.

\section{REFERENCES}

1. Daruich A, et al. Central serous chorioretinopathy: recent findings and new physiopathology hypothesis. Prog Retin Eye Res. 2015;48:82-118.

2. Iacono $\mathrm{P}$, Battaglia Parodi $\mathrm{M}$, Falcomatà $\mathrm{B}$, Bandello F. Central serous chorioretinopathy treatments: a mini review. Ophthalmic Res. 2015;55(2):76-83.

3. van Rijssen TJ, et al. Central serous chorioretinopathy: towards an evidence-based treatment guideline. Prog Retin Eye Res. 2019;73:100770. 
4. Zucchiatti I, et al. Eplerenone versus observation in the treatment of acute central serous chorioretinopathy: a retrospective controlled study. Ophthalmol Ther. 2018;7(1):109-18.

5. Zola M, Daruich A, Matet A, Mantel I, Behar-Cohen F. Two-year follow-up of mineralocorticoid receptor antagonists for chronic central serous chorioretinopathy. $\mathrm{Br}$ J Ophthalmol. 2019;103(8): 1184-9.

6. Bousquet E, et al. Predictive factors of response to mineralocorticoid receptor antagonists in nonresolving central serous chorioretinopathy. Am J Ophthalmol. 2019;201:86-7.

7. Park W, Kim M, Kim RY, Park YH. Comparing effects of photodynamic therapy in central serous chorioretinopathy: full-dose versus half-dose versus half-dose-half-fluence. Graefes Arch Clin Exp Ophthalmol. 2019;257(10):2155-61.

8. Iwase $\mathrm{T}$, Yokouchi $\mathrm{H}$, Kitahashi M, Kubota-Taniai M, Baba T, Yamamoto S. Long-term effects of halftime photodynamic therapy on retinal sensitivity in eyes with chronic central serous chorioretinopathy. Biomed Res Int. 2020;2020:3190136.

9. Chatziralli I, Vlachodimitropoulou A, Daoula C, Vrettou C, Galani E, Theodossiadis G, Theodossiadis $\mathrm{P}$. Eplerenone in the treatment of central serous chorioretinopathy: a review of the literature. Int J Retina Vitreous. 2018;4:33.

10. Karagiannis D, Parikakis E, Kontomichos L, Batsos $\mathrm{G}$, Chatziralli I. The effect of eplerenone in chronic central serous chorioretinopathy refractory to photodynamic therapy. Semin Ophthalmol. 2019;34(6):436-41.

11. Lotery A, et al. Eplerenone for chronic central serous chorioretinopathy in patients with active, previously untreated disease for more than 4 months
(VICI): a randomised, double-blind, placebo-controlled trial. Lancet. 2020;395(10220):294-303.

12. Rabiolo A, Bandello F. Eplerenone is not superior to placebo for chronic central serous chorioretinopathy. Lancet. 2020;395(10220):252-3.

13. Arrigo A, et al. Acute central serous chorioretinopathy subtypes as assessed by multimodal imaging. TVST. 2020. [Epub ahead of print]

14. Sacconi R, Borrelli E, Querques G. Eplerenone for chronic central serous chorioretinopathy. Lancet. 2020;396(10262):1556.

15. Akaike H. Information theory and an extension of maximum likelihood principle. In: Petrov BN, Csaki $\mathrm{F}$, editors. Second international symposium on information theory. AkademiaiKiado: Budapest; 1973. p. 267-81.

16. Safavian SR, Landgrebe D. A survey of decision tree classifier methodology. IEEE Trans Syst Man Cybernet. 1991;21(3):660-74.

17. R Core Team. R: A language and environment for statistical computing. R Foundation for Statistical Computing, Vienna, Austria. 2018. URL https:// www.R-project.org/.

18. Loo RH, et al. Factors associated with reduced visual acuity during long-term follow-up of patients with idiopathic central serous chorioretinopathy. Retina. 2002;22(1):19-24.

19. Lee GI, et al. Risk factors and outcomes of choroidal neovascularization secondary to central serous chorioretinopathy. Sci Rep. 2019;9(1):3927.

20. Chhablani J, et al. Outcomes of treatment of choroidal neovascularization associated with central serous chorioretinopathy with intravitreal antiangiogenic agents. Retina. 2015;35(12):2489-97. 